\title{
Towards an efficient nanophotonic platform integrating quantum memories and single qubits based on rare-earth ions
}

\author{
Tian Zhong, Jonathan M. Kindem, John G. Bartholomew, Jake Rochman, Ioana Craiciu, \\ Evan Miyazono, Andrei Faraon ${ }^{1}$

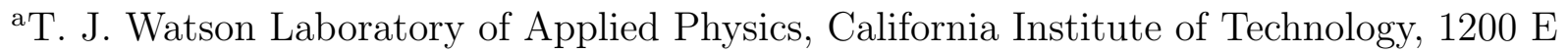 \\ California Blvd, Pasadena, CA, 91125, USA
}

\begin{abstract}
The integration of rare-earth ions in an on-chip photonic platform would enable quantum repeaters and scalable quantum networks. While ensemble-based quantum memories have been routinely realized, implementing single rare-earth ion qubit remains an outstanding challenge due to its weak photoluminescence. Here we demonstrate a nanophotonic platform consisting of yttrium vanadate (YVO) photonic crystal nanobeam resonators coupled to a spectrally dilute ensemble of $\mathrm{Nd}$ ions. The cavity acts as a memory when prepared with spectral hole burning, meanwhile it permits addressing of single ions when high-resolution spectroscopy is employed. For quantum memory, atomic frequency comb (AFC) protocol was implemented in a $50 \mathrm{ppm}$ Nd:YVO nanocavity cooled to $480 \mathrm{mk}$. The high-fidelity quantum storage of time-bin qubits is demonstrated with a $80 \%$ efficient WSi superconducting nanowire single photon detector (SNSPD). The small mode volume of the cavity results in a peak atomic spectral density of $¡ 10$ ions per homogeneous linewidth, suitable for probing single ions when detuned from the center of the inhomogeneous distribution. The high-cooperativity coupling of a single ion yields a strong signature $(20 \%)$ in the cavity reflection spectrum, which could be detected by our efficient SNSPD. We estimate a signal-to-noise ratio exceeding 10 for addressing a single $\mathrm{Nd}$ ion with its $879.7 \mathrm{~nm}$ transition. This, combines with the AFC memory, constitutes a promising platform for preparation, storage and detection of rare-earth qubits on the same ship.
\end{abstract}

Keywords: Quantum optics, Quantum information and processing, Quantum memories

\section{INTRODUCTION}

Quantum optical networks consist of nodes in which entangled quantum states are generated, stored, and processed locally. An integrated platform comprising qubit preparation, memories, and detection on the same chip-scale platform will enable scalable quantum networks for efficient long-distance quantum communications and interconnection of future quantum computers. To date, nano-scale waveguide integrated single or entangled photon sources, superconducting single photon detectors, and passive/active photonic rounting circuits have been demonstrated. Enabling quantum optical storage on-chip will complete a full set of key functionalities required for an integrated quantum repeater node. Furthermore, the potential for generating memory-compatible single photons within the same platform opens a path for efficient quantum repeaters by eliminating freuqncy and bandwidth mismatch that typically limits the quantum light-matter interface efficiency.

Scalable and robust quantum repeater node can be built from quantum light emitters that have long spin coherence times and coherent optical transitions. ${ }^{1}$ Furthermore, in integrated optical quantum networks, these emitters need to be coupled to optical resonators that confine the photons in a single mode and further couple them into optical fibres. The solid-state emitters intensively investigated so far for on-chip quantum network are semiconductor quantum dots (QDs), ${ }^{2}$ color centers (e.g. nitrogen vacancy (NV), SiV) in diamond. ${ }^{3}$ To date, complete quantum control of single QD and NV spins have been realized. Both QDs and NVs ${ }^{4}$ have been coupled to optical nano-cavities. However, the challenge in growing optically identical QDs limits their usability for a

Further author information: (Send correspondence to A. F. and T. Z. )

A. F. E-mail: faraon@caltech.edu

T. Z. E-mail: tzhong@caltech.edu

Advances in Photonics of Quantum Computing, Memory, and Communication X, edited by Zameer UI Hasan, Philip R. Hemmer, Hwang Lee, Alan L. Migdall, Proc. of SPIE Vol. 10118, 101180N

(C) 2017 SPIE · CCC code: $0277-786 X / 17 / \$ 18 \cdot$ doi: $10.1117 / 12.2251673$

Proc. of SPIE Vol. 10118101180 N-1 
scalable architecture. NVs embedded in nanostructures have long electronic spin coherence times ${ }^{3}$ but suffer from optical spectral instabilities such as blinking and spectral diffusion. ${ }^{5}$ These spectral instabilities have made it difficult to construct coherent and efficient optical memories with QDs or NV centers in nano-resonators.

Rare-earth-ions doped in host crystals at cryogenic temperatures exhibit highly coherent spin states in the 4f orbital. ${ }^{6}$ The hyperfine states of REIs can have spin coherence times as long as hours, ${ }^{7}$ the longest ever demonstrated in a solid. These states are connected via optical transitions with the narrowest linewidth in the solid state $(\mathrm{kHz})$ and small inhomogeneous broadening $(\mathrm{MHz}$ to $\mathrm{GHz}) .{ }^{8}$ This outstanding optical and spin coherence makes REI-doped crystals the state-of-the-art material for macroscopic solid-state optical quantum memories. ${ }^{9,10}$ Detection of single REI spins has been recently demonstrated in bulk material, but not using the transitions employed in optical quantum memories. ${ }^{11,12}$

The integration of rare-earth ions in an on-chip quantum nanophotonic platform would enable multiplexed quantum repeaters and scalable quantum networks where REI ensembles act as quantum memories and single REIs act as qubits. ${ }^{13,20}$ Here we demonstrate optical quantum storage in a Rare-earth-doped Yttrium vanadate $\mathrm{YVO}_{4}$ (YVO) photonic crystal nano-beam resonator with small mode volume of 0.8 cubic wavelengths. The unity cooperativity coupling of the $880 \mathrm{~nm}{ }^{4} \mathrm{I}_{9 / 2}{ }^{4} \mathrm{~F}_{3 / 2}$ transition of Neodymium $\left(\mathrm{Nd}^{3+}\right)$ ions to the nano-resonator fulfills cavity impedance matching condition, which facilitates high efficiency quantum storage. ${ }^{14}$ We also show enhanced optical pumping and the realization of an atomic frequency comb (AFC) quantum memory. ${ }^{15}$ In the same device, a low ion density detuned from the center of the inhomogeneous ensemble permits optical detection of resolved individual rare-earth spins. Combining AFC quantum storage and single spin detection, this highly miniaturized platform paves the way for fully integrated quantum nodes and scalable quantum networks.

\section{DESIGN AND FABRICATION OF ONE-SIDED YVO NANOCAVITIES}

We fabricated triangular nano-beam resonator in YVO using focused ion beam milling. ${ }^{16-19,21-23}$ The triangular nanobeam has a width of $690 \mathrm{~nm}$. 31 (11 on one side of the cavity defect mode and 20 on the other side) periodic subwavelength grooves of $147 \mathrm{~nm}$ along the beam axis were milled on top of the nanobeam. The period of the grooves were modulated quadratically over 14 grooves to form defect modes in the photonic bandgap. ${ }^{17}$ The fundamental TM mode, with top, side views shown in Fig. 1c, is chosen because it aligns with the strongest $\mathrm{Nd}$ dipole of the $879.8 \mathrm{~nm}$ transition along c axis of YVO crystals. ${ }^{24,25}$ The theoretical quality factor is $2 \times 10^{5}$ with a mode volume of $0.8(\lambda / \mathrm{n})^{3}$. Coupling from free-space to the nanobeam waveguide was realized by a $45^{\circ}$-angled reflector milled into the sample surface. The one-way coupling efficiency was determined to be $27 \%$ by comparing the cavity-reflected power at a off resonance wavelength (in the photonic bandgap) to that from a flat surface of known reflectivity.

The optimization procedure for an one-sided cavity was started by fabricating symmetric two-sided nanocavities with sufficiently long mirrors (20 grooves) on each side of the cavity mode. The measured $\mathrm{Q}$ was in the range of 20,000 to 40,000, corresponding to an intrinsic loss (likely due to surface scattering) rate $\kappa_{\mathrm{sc}}=9-18 \mathrm{GHz}$. Then the number of grooves on one side was decremented from 20 to 11, and the corresponding Q decreased from 20,000 to $3,800(\kappa=90 \mathrm{GHz}$ ). The final device (with 11 grooves) is expected to be dominantly one-sided and over-critically coupled with the waveguide: $\kappa_{\text {in }} / \kappa=0.8$. In reality, the measured reflection spectrum in Fig. 2 shows a near critical coupling to the waveguide with $\kappa_{\text {in }} \sim 0.5 \kappa=45 \mathrm{GHz}$. The deviation from the design might be due to additional fabrication imperfections when making highly asymmetric groove patterns, which could be improved in future fabrication processes.

\section{IMPEDANCE-MATCHED QUANTUM LIGHT-MATTER INTERFACE}

Unlike macroscopic bulk memories that require long crystals, thus high optical depth, to achieve high storage efficiency, memories based on an impedance-matched cavity can efficiently map a photon to a small ensemble of atoms that are coupled to a cavity with a unity collective cooperativity $C=4 N g^{2} / \kappa \Gamma_{h}$ where $g$ is the atomcavity coupling rate, $\Gamma_{h}$ is the atomic homogeneous linewidth, and $N$ is the atomic density per $\Gamma_{h}$. Figure 2 plots the reflection spectrum of the cavity on resonance with the $\mathrm{Nd}$ ensemble. The center peak is a result of coupling between the cavity field and the Nd dipoles of the ${ }^{4} F_{3 / 2}\left(Z_{1}\right)-{ }^{4} I_{9 / 2}\left(Y_{1}\right)$ transition at $879.7 \mathrm{~nm}$. The contrast of the peak reflects the averaged Rabi frequency $\bar{g}$ experienced by the ions that are randomly

Proc. of SPIE Vol. 10118 101180N-2 

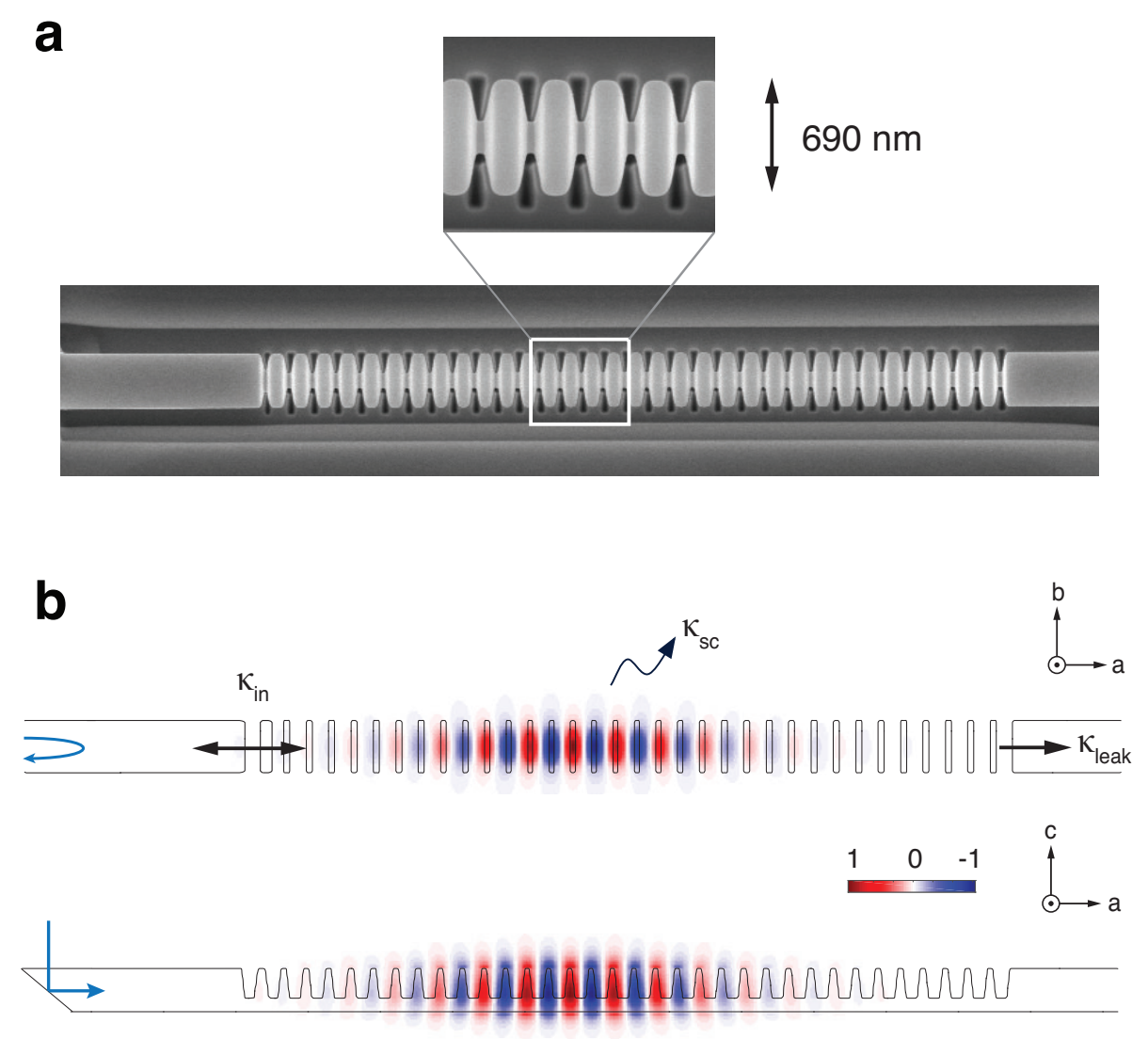

Figure 1. (a) Scanning electron microscope image of the Nd:YVO nano-beam resonator device. ${ }^{16,17}$ The device was fabricated using focused ion beam milling. The photonic bandgap is formed by periodic groove-shaped structures on a trianglar nanobeam with a width of $690 \mathrm{~nm}$. (b) Schematics of the nano-beam resonator with simulated field profiles of the fundamental TM mode. The TM polarization aligns with the c axis of the YVO crystal. One (left) side of the photonic crystal mirror (grooves) was shortened to allow over-critical coupling between the waveguide and the cavity. The theoretical Q of the one-sided cavity is $1 \times 10^{5}$, and measured $\mathrm{Q}$ of the fabricated device is 3900 .

distributed in the cavity, and gives an effective $C \sim 0.8$ at the center of the ensemble frequency. The one-sided cavity design combined with the near unity coupling cooperativity leads to a quantum storage efficiency (the probability for an input photon to be mapped onto an atomic excitation) of $\sim 50 \%$ in our nanocavity device. In comparison, achieving the same storage efficiency in bulk (assuming the same doping concentration) would require a crystal length of centimeters. Further optimization of the device fabrication can in principle attain over-critical waveguide-cavity coupling of $\kappa_{i n} / \kappa=0.9$, and exact impedance matching condition can be realized with slightly increased Q. These improvements would bring the storage efficiency to $90 \%$, even though the size of the rare-earth ensemble remains small (on the order of 10 ions per $\Gamma_{h}$ ).

We further characterized the cavity enhancement effect by measuring the photoluminescence (PL) lifetime (optical $\mathrm{T}_{1}$ ) in the cavity. We observed a $1 / e$ decay time of $4.5 \mu$ s that is 20 times faster than that from the bulk $(90 \mu \mathrm{s})$. This Purcell enhancement increased the $Y_{1}-Z_{1}$ branching ratio from initially $27 \%^{13}$ to $\sim 97 \%$, which effectively created an efficient $\Lambda$ system for optical pumping experiments required for AFC memory preparation. In particular, the Purcell enhanced spontaneous decay rate of the non-spin preserving transition created a condition for increased optical pumping efficiency for ions in the cavity. This cavity-enhanced optical pumping highlights a unique feature of the nanophotonic device for optical quantum memory applications. 


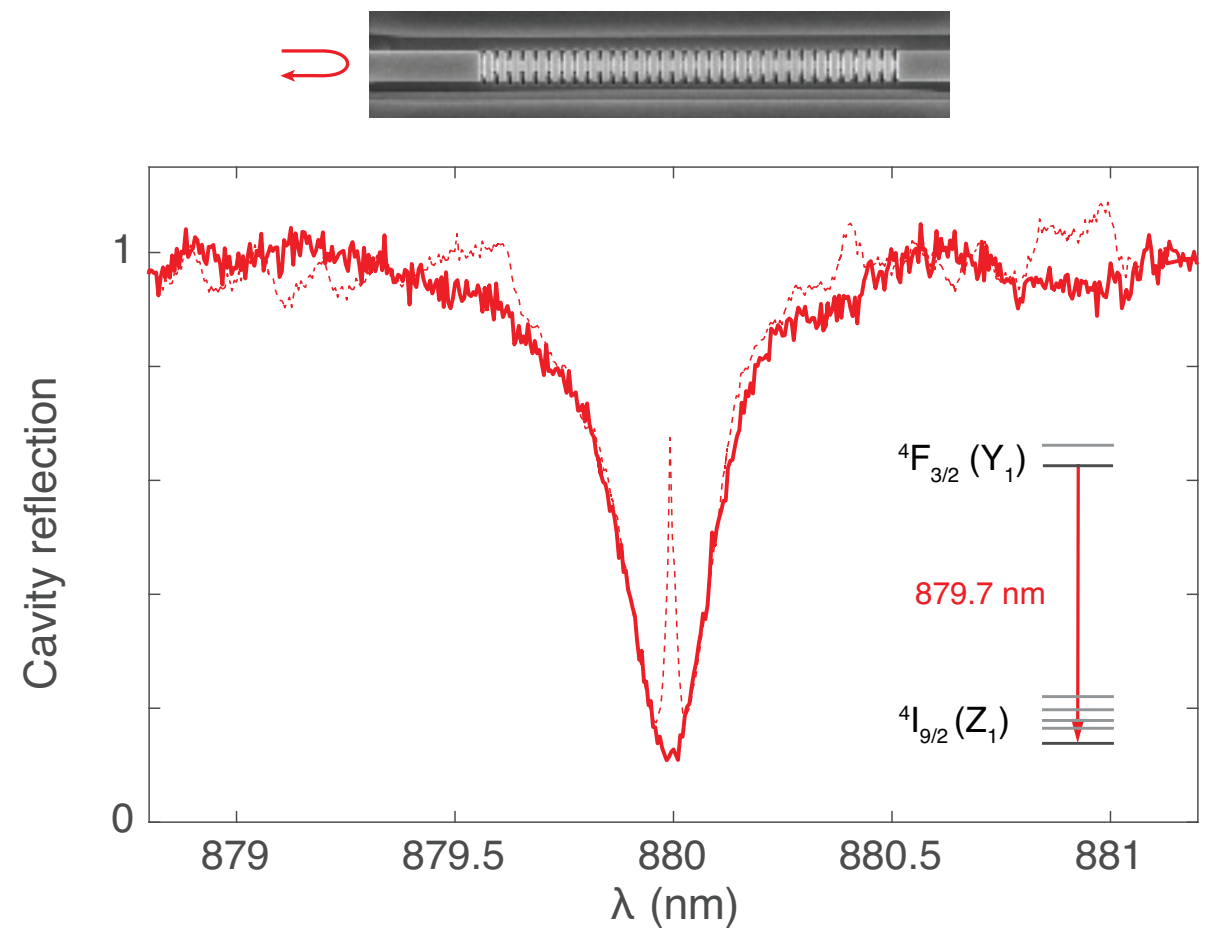

Figure 2. Reflection spectrum of the Nd:YVO nanocavity measured from one (left) side of the nanobeam waveguide. The waveguide-cavity coupling is near critical as indicated by an almost full extinction of the reflection at the cavity resonance. When the cavity is tuned on resonance with the $879.7 \mathrm{~nm}$ transition of $\mathrm{Nd}$ ions at a cryogenic temperature, a peak appeared at the atomic resonance indictating coherent coupling between the cavity with the Nd ensemble. The collective coupling cooperativity was 0.8 , calculated from the contrast of the peak with respect to the cavity reflection dip.

\section{QUANTUM STORAGE BASED ON ATOMIC FREQUENCY COMBS}

With efficient optical pumping, we demonstrate nanophotonic AFC quantum storage by creating a comb in the ensemble's inhomogeneous absorption profile. The AFC was generated by sending a sequence of 10-ns pulse pairs separated by a time interval $t=1 / \Delta$ where $\Delta$ is the frequency spacing of the comb. The pulse sequence was optimized for best echo efficiencies for a given $t$. We prepared an AFC with a finesse $\mathrm{F}=3.3$ and $\delta=13.3 \mathrm{MHz}$ with a nearly vanishing absorption background. After a wait time of $200 \mu \mathrm{s}$, an input photon from attenuated laser pulse was sent to the nanocavity and an AFC echo was retrieved after a delay time $t=75$ ns. Figure 3 plots the reflected input and the AFC echo signal for a coherent photon, with a mean photon number of 0.58 . The input photon number was determined from a cavity reflected signal that was 1-nm detuned from the cavity resonance. The AFC device efficiency, defined as the ratio of echo to input photons, was measured at $\sim 1 \%$ at a wait time of $200 \mu \mathrm{s}$.

\section{OPTICALLY DETECTABLE SINGLE RARE-EARTH IONS IN A NANOCAVITY}

Two-pulse photon echo experiments were performed to extract optical coherence time $\mathrm{T}_{2}$ of individual $\mathrm{Nd}$ ions in the cavity. We measured $\mathrm{T}_{2} \sim 3.0 \mu \mathrm{s}$ for the coupled ions in the nano-resonator at $0.5 \mathrm{~K}$ temperature with $340 \mathrm{mT}$ applied magnetic field along the cystal c axis, which agrees well with the $\mathrm{T}_{2}=3.2 \mu \mathrm{s}$ measured in the bulk sample under the same temperature and magnetic field condition. With an inhomogeneous broadening of $2 \mathrm{GHz}$, our nanocavity contains a total of $\sim 4 \times 10^{4}$ ions with a peak spectral density of $\sim 10$ ions per $\Gamma_{h}$, where $\Gamma_{h}=1 / \pi / T_{2}=100 \mathrm{kHz}$. The cooperativity of a single ion positioned at the cavity field maximum will be 0.4 . This corresponds to a strong peak in the cavity reflection spectrum, which could be detected by our low-dark count SNSPD detector. We estimate a signal-to-noise ratio exceeding 10 when directly scanning the 


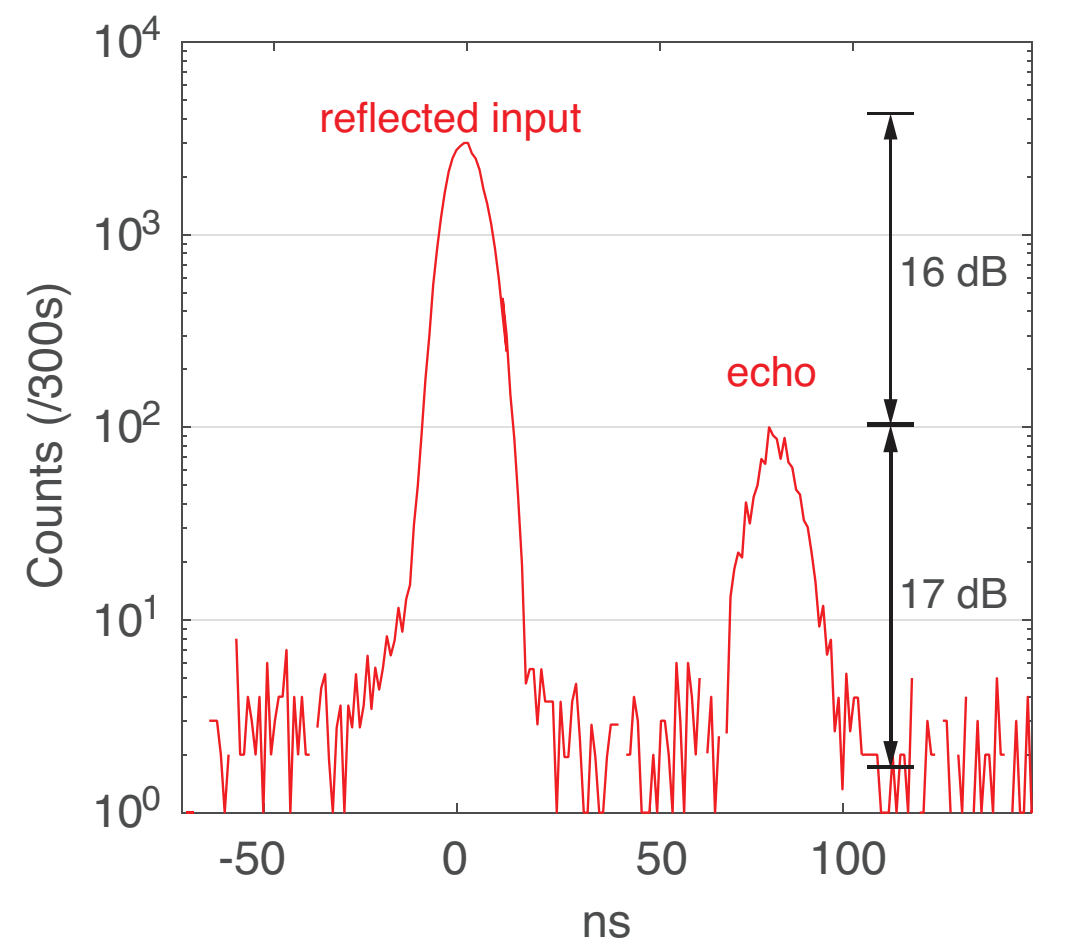

Figure 3. Nanophotonic AFC quantum storage in a Nd:YVO nanocavity. The reflected input signal of weak coherent pulse (mean photon number was 0.58) and the retrieval AFC echo signal is plotted. The delay time between the input and echo photons was $75 \mathrm{~ns}$. The echo signal, integrated for $300 \mathrm{~s}$, is $17 \mathrm{~dB}$ higher than the average background noise, indicating high-fidelity quantum storage as a result of efficient optical pumping.

on-resonance reflection by single ions in the cavity. This cavity-assisted scheme of addressing single rare-earth ions can find applications in studying long-range ion-ion interactions and non-destructive photon detections ${ }^{26}$ a critical functionality for an integrated quantum repeaternetwork.

\section{ACKNOWLEDGMENTS}

This work was funded by California Institute of Technology (Caltech) and National Science Foundation (NSF) CAREER award number 1454607. Equipment funding was also provided by the Institute of Quantum Information and Matter (IQIM), an NSF Physics Frontiers Center with support of the Moore Foundation. The device nanofabrication was performed in the Kavli Nanoscience Institute at Caltech.

\section{REFERENCES}

[1] Jiang, L., Taylor, J. M., \& Lukin, M. D. Fast and robust approach to long-distance quantum communication with atomic ensembles. Phys. Rev. A A, 012301 (2007).

[2] Michler, P. (Ed.) Single Quantum Dots (Springer Berlin Heidelberg, 2009).

[3] Aharonovich, I., and Neu, E. "Diamond Nanophotonics." Adv. Optical Mater. 2, 911-928 (2014).

[4] Faraon, A., Barclay, P. E., Santori, C., Fu, K. C., and Beausoleil, R. G. "Resonant enhancement of the zero-phonon emission from a colour centre in a diamond cavity." Nature Photon. 5, 301-305 (2011).

[5] Faraon., A., Santori, C., Huang, Z., Acosta, V. M., and Beausoleil, R. G. "Coupling of Nitrogen-Vacancy Centers to Photonic Crystal Cavities in Monocrystalline Diamond." Phys. Rev. Lett. 109, 033604 (2012).

[6] Thiel, C., Böttger, T., and Cone, R. "Rare-earth-doped materials for applications in quantum information storage and signal processing." J. Luminesc. 131, 353-361(2001). 
[7] Zhong, M., Hedges, M. P., Ahlefeldt, R. L., Bartholomew, J. G., Beavan, S. E., Wittig, S. M., Longdell, J. J., and Sellars, M. J. "Optically addressable nuclear spins in a solid with a six-hour coherence time." Nature 517, 177-180 (2015).

[8] Sun, Y., Thiel, C. W., Cone, R. L., Equall, R. W. and Hutcheson, R. L. "Recent progress in developing new rare earth materials for hole burning and coherent transient applications." J. Lumin. 98, 281-287 (2002).

[9] Tittel, W., Afzelius, M., Chaneliére, T., Cone, R. L., Kröll, S., Moiseev, S. A., and Sellars, M. "Photon-echo quantum memory in solid state systems." Laser \& Photon. Rev. 4, 244-267 (2010).

[10] de Riedmatten, H., Afzelius, M., Staudt, M. U., Simon, C., and Gisin, N. "A solid-state light-matter interface at the single-photon level." Nature 456, 773-777 (2008).

[11] Kolesov R. et al. "Optical detection of a single rare-earth ion in a crystal." Nature Commun. 3, 1029 (2012).

[12] Utikal, T. et al. "Spectroscopic detection and state preparation of a single praseodymium ion in a crystal." Nature Commun. 5, 3627 (2014).

[13] McAuslan, D. L., and Longdell, J. J. "Cavity QED using rare-earth-metal-ion dopants in monolithic resonators: What you can do with a weak oscillator." Phys. Rev. A 80, 062307 (2009).

[14] Afzelius, M., and Simon, C. "Impedance-matched cavity quantum memory." Phys. Rev. A 82, 022310 (2010).

[15] Afzelius, M. et al. Demonstration of Atomic Frequency Comb Memory for Light with Spin-Wave Storage. Phys. Rev. Lett. 104, 040503 (2010).

[16] Zhong, T., Kindem, J., Miyazono, E., and Faraon, A. "Nanophotonic coherent light-matter interfaces based on rare-earth doped crystals." Nat. Commun. 6, 8206 (2015).

[17] Zhong, T., Rochman, J., Kindem, J., Miyazono, E., and Faraon, A. "High quality factor nanophotonic resonators in bulk rare-earth doped crystals." Opt. Express 24, 536-544 (2016).

[18] Zhong, T., Hartz, A., Miyazono, E., and Faraon, A. "Towards Coupling Rare Earth Ions to a $\mathrm{Y}_{2} \mathrm{SiO}_{5}$ Nanophotonic Resonator." CLEO: 2014 OSA Technical Digest (online), paper FTh1B.5 (Optical Society of America, 2014).

[19] Zhong, T., Kindem, J., Miyazono, E., and Faraon, A. "Nanophotonic Quantum Memory Based on RareEarth-Ions Coupled to an Optical Resonator." CLEO: 2015 OSA Technical Digest (online), paper FW3A.3 (Optical Society of America, 2015).

[20] Zhong, T., Kindem, J., Miyazono, E., and Faraon, A. "Towards Detection of Single Rare-Earth-Ions in a Nanophotonic Resonator." CLEO: 2015 OSA Technical Digest (online), paper FTh4B.7 (Optical Society of America, 2015).

[21] Zhong, T., Kindem, J., Miyazono, E., and Faraon, A. "Nanophotonic photon echo memory based on rareearth-doped crystals." APS Meeting Abstracts 1, 37003( 2015).

[22] Miyazono, E., Zhong, T., Craiciu, I., Kindem, J., and Faraon, A. "Coupling of erbium dopants to yttrium orthosilicate photonic crystal cavities for on-chip optical quantum memories." Appl. Phys. Lett. 108, 01111 (2016).

[23] Zhong, T., Kindem, J. M., Rochman, J., Miyazono, E., Faraon, A., Ferrier, A., and Goldner, P. "On-chip quantum storage in a rare-earth-doped photonic nanocavity." Proc. SPIE 9762, Advances in Photonics of Quantum Computing, Memory, and Communication IX, 97620J (2016).

[24] Zhong, T., Kindem, J., Rochman, J., and Faraon, A., "50 GHz quantum photonic storage in a cavityprotected rare-earth ensemble." CLEO: 2016 OSA Technical Digest (online), paper FM4C. 2 (Optical Society of America, 2016).

[25] Zhong, T., Kindem, J., Rochman, J., and Faraon, A. "Interfacing broadband photonic qubits to on-chip cavity-protected rare-earth ensemble." Nat. Commun. 7, 14107 (2016).

[26] O'Brien, C., Zhong, T., Faraon, A., and Simon, C., "Nondestructive photon detection using a single rareearth ion coupled to a photonic cavity." Phys. Rev. A 94, 043807 (2016). 